VOL. $10(1974), 51-58$.

\title{
A class of majorant functions for contractors and equations
}

\section{Mieczyslaw Altman}

\begin{abstract}
Majorant functions for contractors can be defined in a natural way. Such a case is considered here in order to find iterative solutions of general equations in Banach spaces by means of contractors. A class of majorant functions is defined which contains in particular the linear majorant ones. Local and global existence and convergence theorems are proved.
\end{abstract}

\section{Natural majorant functions}

Let $P: D \subset X \rightarrow Y$ be a non-linear operator with domain $D$ containing a sphere $S=S\left(x_{0}, r\right)$ with radius $r$ and centre $x_{0}, X$ and $Y$ being Banach spaces. Denote by $L(Y \rightarrow X)$ the space of all linear bounded operators from $Y$ into $X$ and let $\Gamma: D \rightarrow L(Y \rightarrow X)$ be a mapping, that is, for fixed $x \in D, \Gamma(x): Y \rightarrow X$ is a bounded linear operator from $Y$ into $X$. Put

(1.1) $Q(s)=\max \{\|P(x+\Gamma(x) y)-P x-y\| \mid x \in S, y \in Y, x+\Gamma(x) y \in D,\|y\| \leq s\}$, assuming that $Q(s)$ is finite for $0<s \leq n$, where $\eta>0$ is a certain. number to be defined below. It follows from (1.1) that $Q(0)=0$ and $Q(s)$ is non-decreasing. It results also from (1.1) that

$$
\|P(x+\Gamma(x) y)-P x-y\| \leq Q(\|y\|)
$$

for $x \in S, y \in Y$ whenever $x+\Gamma(x) y \in D$. If there exists a function $Q$ satisfying (1.2), then we say that $\Gamma$ is a contractor for $P$ rith majorant function $Q$ satisfying the contractor inequality (1.2). Thus, a

Received 28 August 1973. 
majorant function for $\Gamma$, if any, can be defined in a natural way by (1.1).

Consider now the problem of solving the operator equation

$$
P x=0, \quad x \in D \text {. }
$$

We assume that $x_{0}$ is chosen so as to satisfy

$$
\left\|P x_{0}\right\| \leq n
$$

and $\Gamma$ is bounded; that is

$$
\|\Gamma(x) y\| \leq B \text { for } x \in S .
$$

In addition, we suppose that

$$
Q(8)<8 \text { for } 8>0
$$

and there exists the integral

$$
I(\eta)=\int_{0}^{n} s[s-Q(s)]^{-1} d s<\infty
$$

provided that the function $s /(s-Q(s))$ is non-increasing.

In order to solve equation (1.3) we use the following iterative procedure

$$
x_{n+1}=x_{n}-\Gamma\left(x_{n}\right) P x_{n}, n=0,1, \ldots .
$$

Simultaneously we consider the following numerical iterative procedure

$$
s_{n+1}=Q\left(s_{n}\right), s_{0}=n, n=0,1, \ldots,
$$

where $Q$ is the majorant function for $\Gamma$ defined by (1.1) or (1.2).

An operator $P$ is said to be closed if $x_{n} \in D, x_{n} \rightarrow x$ and $P x_{n}+y$ imply $x \in D$ and $y=P x$.

LEMMA 1.1. Let $Q(s)>0$ for $s>0$ with $Q(0)=0$ be a frunction satisfying conditions (1.6) and (1.7), where $s /(s-Q(s))$ is nonincreasing. Then the series $\sum_{i=0}^{\infty}{ }^{s} i$ is convergent, where the sequence $\left\{s_{n}\right\}$ is defined by (1.9) and the following estimate holds: 
(1.10)

$$
\sum_{i=n}^{\infty} s_{i} \leq \sum_{i=n}^{\infty} \int_{s_{i+1}}^{s} s[s-Q(s)]^{-1} d s=\int_{0}^{s} n s[s-Q(s)]^{-1} d s .
$$

Proof. We have for $m>n$, by (1.9),

$$
\begin{aligned}
\sum_{i=n}^{m-1} s_{i} & =\sum_{i=n}^{m-1} s_{i}\left(s_{i}^{-8}{ }_{i+1}\right) /\left(s_{i}-Q\left(s_{i}\right)\right) \leq \sum_{i=n}^{m-1} \int_{s_{i+1}}^{s} s[s-Q(s)]^{-1} d s \\
& =\int_{s_{m}}^{n} s[s-Q(s)]^{-1} d s
\end{aligned}
$$

since the function $s /(s-Q(8))$ is non-increasing by assumption.

Inequality (1.10) follows from (1.11), and the convergence of the series $\sum_{i=0}^{\infty} s_{i}$ results from $(1.7)$, and we have

$$
\sum_{i=0}^{\infty} \boldsymbol{s}_{i} \leq I(n)
$$

Lemma 1.1 will be applied to prove the following.

THEOREM 1.1. Let $P: D+Y$ be a closed non-linear operator with domain $D$ containing the sphere $S$. Suppose that $\Gamma$ is a contractor for $P$, and satisfies condition (1.5) and the contractor inequality (1.2).

Furthermore, assume that the majorant fronction $Q$ satisfies conditions (1.6) and (1.7), where $n$ is given by (1.4). Finally let

$$
B I(n)=r \text {. }
$$

Then all $x_{n}$ lie in $S$ and the sequence $\left\{x_{n}\right\}$ defined by (1.8) converges to a solution $x$ of equation (1.3) and the error estimate is as follows:

$$
\left\|x-x_{n}\right\| \leq B \int_{0}^{s} n[s-Q(s)]^{-1} d s .
$$

Proof. It follows from the contractor inequality (1.2) with $x=x_{n}$ and $y=-P x_{n}$ that

$$
\left\|P x_{n+1}\right\| \leq Q\left(\left\|P x_{n}\right\|\right)
$$


Since $Q$ is non-decreasing we prove by induction, in virtue of (1.15), that

$$
\left\|P x_{n+1}\right\| \leq Q\left(s_{n}\right)=s_{n+1}, n=0,1, \ldots
$$

It follows from $(1.8),(1.5)$ and $(1.16)$, by using induction, that

$$
\left\|x_{n+1}-x_{n}\right\| \leq B s_{n}, n=0,1, \ldots .
$$

Hence it follows, in virtue of Lemma 1.1, in virtue of (1.11), that

$$
\left\|x_{m}-x_{n}\right\| \leq B \sum_{i=n}^{m-1} s_{i} \leq B \int_{s_{m}}^{s} n[s-Q(s)]^{-1} d s
$$

and

(1.18) $\quad\left\|x_{n}-x_{0}\right\| \leq B \sum_{i=0}^{n-1} s_{i} \leq B \int_{s_{n}}^{\eta} s[s-Q(s)]^{-1} d s<B I(\eta)=r$,

in virtue of (1.13). Thus, it results from (1.18) that $x_{n} \in S$ for $n=0,1, \ldots$ and $(1.17)$ shows that the sequence $\left\{x_{n}\right\}$ converges to some element $x$. On the other hand, by (1.12), the series $\sum_{n=0}^{\infty} s_{n}$ is convergent and obviously $s_{n}+0$ as $n \rightarrow \infty$. Therefore, (1.16) implies that $P x_{n} \rightarrow 0$ as $n \rightarrow \infty$. Since $P$ is closed, it follows that $P x=0$. The error estimate (1.14) follows from (1.17) by letting $m \rightarrow \infty$ so that $s_{m} \rightarrow 0$, and the proof is complete.

It is easily seen that the case of a contractor with linear majorant function, investigated in [1], is a particular one of Theorem 1.1. In other words, we have the following.

COROLLARY 1.1. Under the corresponding hypotheses of Theorem 1.1 suppose that the majorant function $Q$ is defined by $Q(s)=q$ s with $0<q<1$. Then all assertions of Theorem 1.1 hold true and the error estimate (1.14) yields

$$
\left\|x-x_{n}\right\| \leq B n q^{n} /(1-q) .
$$


Proof. Condition $(1.6)$ is obviously fulfilled and $Q(0)=0$. The function $Q$ is evidently increasing. Since the function $s /(s-Q(s))=1 /(1-q)$ is constant, $I(\eta)=\eta /(1-q)<\infty$, yielding condition (1.7). In virtue of (1.9), we have

$$
s_{n}=n q^{n}, n=0,1, \ldots .
$$

Hence, it follows that in this particular case the error estimate (1.14) coincides with (1.19) and condition (1.13) is replaced by $B \eta /(1-q)=r$.

EXAMPLE. Let $F: S \rightarrow S \subset X$ be a contraction with Lipschitz constant $q<1$, that is

$$
\|F x-F \bar{x}\| \leq q\|x-\bar{x}\| \text { for all } x, \bar{x} \in S \text {. }
$$

Put $P x=x-F x$. Then it is easy to verify-that $\Gamma(x) \equiv I$. (the identity mapping of $X$ ) is a contractor for $P$ with majorant function $Q$ defined by $Q(s)=q s$.

Thus, Theorem 1.1 as well as Corollary 1.1 generalize the well known local Banach contraction principle.

REMARK 1.1. Suppose that in addition to the hypotheses of Theorem 1.1 the contractor $\Gamma(x)$ maps $Y$ onto $X$. Then the solution of equation (1.3) is unique.

Proof. If $x$ and $\bar{x}$ are two solutions of equation (1.3), then there exists an element $y \in Y$ such that

$$
\bar{x}=x+\Gamma(x) y \text {, since } \Gamma(x) \text { is onto. }
$$

It follows from the contractor inequality (1.2) that $\|y\|<Q(\|y\|)<\|y\|$, if $\|y\|>0$, in virtue of (1.6). Hence, we obtain a contradiction which proves that $\|y\|=0$, that is $\bar{x}=x$.

\section{Global existence and convergence theorem}

Theorem 1.1 yields a local existence and convergence theorem. However, using the same argument one can obtain a global existence and convergence theorem. Let $P: D \subset X \rightarrow Y$ be a non-linear operator and let $\Gamma: D \rightarrow L(Y \rightarrow X)$. We assume that $\Gamma(x)(Y) \subset D$ for all $x \in D$ and that the domain $D$ of $P$ is a linear subset of $X$. Then we can replace (1.1) by 


$$
Q(s)=\max \{\|P(x+\Gamma(x) y)\| \mid x \in D, y \in Y,\|y\| \leq s\}
$$

assuming that $Q(s)$ is finite for arbitrary $s>0$. Then the contractor inequality (1.2) is replaced by the following one:

$$
\|P(x+\Gamma(x) y)-P x-y\| \leq Q(\|y\|)
$$

for $x \in D$ and arbitrary $y \in Y$. Condition (1.5) should be replaced by

$$
\|\Gamma(x)\| \leq B \text { for } x \in D \text {. }
$$

As in Paragraph 1 we assume that the majorant function $Q$ for $\Gamma$ satisfies condition (1.6) and that the function $s /(s-Q(s))$ is nonincreasing for $Q \leq s<\infty$. Finally, we assume that there exists the integral

$$
I(a)=\int_{0}^{a} s[s-Q(s)]^{-1} d s<\infty
$$

for arbitrary positive $\dot{a}$.

Now we can prove the following global existence and convergence.

THEOREM 2.1. Let $P: D \rightarrow Y$ be a closed non-linear operator and let $\Gamma$ be a bounded contractor for $P$ satisfying the contractor inequality (2.2), condition (2.3) and $\Gamma(x)(y) \subset D$ for arbitrary $x \in D$. Let the majorant function $Q$ be non-decreasing and satisfy condition (1.6) and let the junction $s /(s-Q(s))$ be non-increasing for $0 \leq s<\infty$. If the integral (2.4) exists for arbitrary $a>0$, then $P$ maps $D$ onto the whole of $Y$ and. the sequence $\left\{x_{n}\right\}$ defined by

$$
x_{n+1}=x_{n}-\Gamma\left(x_{n}\right)\left[P x_{n}-l\right], n=1,2, \ldots,
$$

where $x_{0}$ is an arbitrary initial approximation, converges to a solution $x$ of $P x-Z=0$, where $Z$ is an arbitrary element of $Y$. The error estimate (1.14) holds true, where the sequence $\left\{s_{n}\right\}$ is defined by (1.9) with $\left\|P x_{0}-2\right\| \leq n=s_{0}$.

Proof. Since for arbitraxy $l \in Y$ the operators defined by $P x$ and $P x-Z$ have the same contractor $\Gamma$, it is sufficient to show that $P x=0$ has a solution $x$ and that the sequence $\left\{x_{n}\right\}$ defined by (2.5) with $Z=0$ converges to $x$. Using the same argument as in the proof of 
Theorem 1.1 we prove $(1.16)$ and $(1.17)$ by induction. In virtue of Lema 1.1 the series $\sum_{n=0}^{\infty} s_{n}$ is convergent. Hence, it follows from (1.17) that the sequence $\left\{x_{n}\right\}$ converges to some element $x$. Then all assertions follow in the same way as in the proof of Theorem 1.1 .

REMARK 2.1. Under the hypotheses of Theorem 2.1 if, in addition $\Gamma(x)$ is onto for every $x \in D$, then $P$ is a one-to-one mapping onto the whole of $y$.

Proof. The proof is the same as that of Remark 1.1 .

COROLLARY 2.1. If the majorant fronction $Q$ in Theorem 2.1 is defined by $Q(s)=q s$ with $0<q<1$, then all assertions of Theorem 2.1 hold true and the error estimate is given by (1.19) where $\left\|P x_{0}-2\right\| \leq n$.

Proof. The proof follows from that of Corollary 1.1.

EXAMPLE. Let $F: X \rightarrow X$ be a contraction with Lipschitz constant $q<1$. Then $\Gamma(x) \equiv I$ is a contractor for $P$ defined by $P x=x-F x$ with majorant function $Q(s)=q s$. Thus, both Theorem 2.1 and Corollary 2.1 generalize the well known global Banach contraction principle. The case of contractors with linear majorant functions is investigated in [1]. : More facts about contractors with non-linear majorant functions are presented in [2].

REMARK 2.2. The class of majorant functions satisfying the hypotheses of Lemma 1.1 contains all linear majorant functions $Q$ defined by $Q(s)=q s$ with $0<q<1$. It is easily seen that majorant functions $Q$ which cannot be majorised by linear ones necessarily possess the following property:

$$
s /(s-Q(s))+\infty \text { as } s \rightarrow 0 \text {. }
$$

In fact, since the function $s /(s-Q(s))$ is non-increasing by assumption it must be bounded by some positive constant $M$, if condition $(\alpha)$ is not satisfied. But $s /(s-Q(s)) \leq M$ implies $Q(s) \leq q s$ with $q=(M-1) / M<1$ and $M>1$ so that $Q$ can be majorized by a linear function with $q<1$.

Let us observe that the error estimate (1.14) is more accurate than the error estimate (1.19) obtained by replacing if possible the majorant function $Q$ by a linear one with $q=(M-1) / M$. 


\title{
References
}

[1] M. Altman, "Inverse differentiability contractors and equations in Banach spaces", Studia Math. 46 (1973), 1-15.

[2] Mieczyslaw Altman, "Contractors with nonlinear majorant functions and equations in Banach spaces", Boll. Un. Mat. Ital. (to appear).

\author{
Department of Mathematics, \\ University of Newcastle, \\ Newcastle, \\ New South Wales \\ and \\ Department of Mathematics, \\ Louisiana State University, \\ Baton Rouge, \\ Louisiana, \\ USA.
}

